\title{
Superfícies de resposta espectro-temporal de imagens do sensor MODIS para classificação de área de soja no Estado do Rio Grande do Sul
}

\author{
Spectral-temporal response surface of MODIS sensor images for soybean area classification \\ in Rio Grande do Sul State
}

\author{
Conrado de Moraes Rudorff ${ }^{\mathrm{I}}$ Rodrigo RizziI ${ }^{I I}$ Bernardo Friedrich Theodor Rudorff ${ }^{\mathrm{II}}$ \\ Luciana Miura Sugawara' Carlos Antônio Oliveira Vieira' ${ }^{\text {III }}$
}

\section{RESUMO}

Este trabalho objetivou avaliar o potencial e as limitações das imagens MODIS para classificação e estimativa de área de soja por meio do método de superfície de resposta espectro-temporal (Spectral-Temporal Response Surface STRS). Um mapa temático das áreas com soja, oriundo da classificação de imagens Landsat do Estado do Rio Grande do Sul, foi utilizado como referência para auxiliar na orientação da amostragem dos pixels de treinamento e para a comparação dos resultados. Seis imagens compostas do sensor MODIS foram utilizadas para a classificação supervisionada da área de soja por meio do algoritmo de máxima verossimilhança (MAXVER) adaptado ao método STRS. Os resultados foram avaliados pelo coeficiente Kappa para a totalidade da área em estudo e também para uma região de latifúndios e outra de minifúndios. O método STRS subestimou em 6,6\% a área de soja para toda a região estudada, sendo que a estatística Kappa foi de 0,503. Para as regiões de latifúndios e minifúndios, a área de soja foi superestimada em 8\% (Kappa=0,424) e subestimada em 43,4\% (Kappa=0,358), respectivamente. As imagens MODIS, por meio do método STRS, demonstraram ter potencial para classificar a área de soja, principalmente em regiões de latifúndios. Em regiões de minifúndios, a correta identificação e classificação das áreas de soja mostrou-se pouco eficiente em razão da baixa resolução espacial das imagens MODIS.

Palavras-chave: sensoriamento remoto agrícola, estimativa de área de culturas agrícolas, imagem de satélite.

\section{ABSTRACT}

This paper was aimed at evaluating the potential and the limitations of MODIS images for soybean classification

\begin{abstract}
and area estimation through a Spectral-Temporal Response Surface (STRS) method. A soybean thematic map from Rio Grande do Sul State, Brazil, derived from Landsat images was used as reference data to assist both sample training and results comparison. Six 16-day composite MODIS images were classified through a supervised maximum likelihood algorithm (MAXVER) adapted to the STRS method. The results were evaluated using the Kappa coefficient for the entire study area and for one region dominated by large farms and another by small ones. The STRS method underestimated the soybean area by $6.6 \%$, for the entire study area, with a Kappa coefficient of 0.503. For regions with large and small farms the soybean area was overestimated by $8 \%($ Kappa $=0.424)$ and underestimated by $43.4 \%$ (Kappa $=0.358)$, respectively. Eventually, MODIS images, through the STRS method, demonstrated good potential to classify and estimate soybean area, mainly in regions with large farms. For regions with small farms the correct identification and classification of soybean areas showed to be less efficient due to the low spatial resolution of MODIS images.
\end{abstract}

Key words: agricultural remote sensing, crop area estimation, satellite image.

\section{INTRODUÇÃO}

A regulação dos estoques e dos preços dos produtos agrícolas depende, em boa parte, da disponibilidade de informações confiáveis a respeito da estimativa da área plantada. Várias pesquisas têm demonstrado que as imagens de sensoriamento remoto oferecem meios para identificar e mapear as grandes

'Divisão de Sensoriamento Remoto (DSR), Instituto Nacional de Pesquisas Espaciais (INPE), Avenida dos Astronautas, 1758, 12.227-010, CP 515, São José dos Campos, SP, Brasil. E-mail: cmr@dsr.inpe.br. Autor para correspondência.

"DSR, INPE, São José dos Campos, SP, Brasil.

IIIDepartamento de Engenharia Civil, Setor de Engenharia de Agrimensura, Universidade Federal de Viçosa (UFV), Viçosa, MG, Brasil. 
culturas a fim de auxiliar na geração de estatísticas agrícolas mais confiáveis (IPPOLITI-RAMILO et al., 2003; RUDORFF et al., 2004; RIZZI \& RUDORFF, 2005).

A discriminação de culturas agrícolas em imagens de sensoriamento remoto geralmente é feita por meio de técnicas de classificação digital em que se utilizam uma ou mais imagens obtidas em períodoschave, quando a resposta espectral da cultura de interesse está bem caracterizada e diferenciada dos demais alvos da cena. Entretanto, é comum observar a presença de alvos com comportamento espectral similar ao da cultura de interesse. Contrariamente, é possível que uma mesma cultura se encontre em diferentes estádios de crescimento (por exemplo, a semeadura em diferentes datas) e, conseqüentemente, apresente padrões espectrais diversos que dificultam a correta identificação da cultura por meio do procedimento de classificação digital. Estas e outras condições podem acarretar erros consideráveis no resultado da classificação. Os erros da classificação digital podem ser corrigidos por meio de uma criteriosa interpretação visual na tela do computador, utilizando o conhecimento do intérprete sobre a cultura e a região de interesse, bem como imagens adquiridas em diferentes datas, dentro de períodos-chave ao longo do seu ciclo (RIZZI \& RUDORFF, 2005; RUDORFF et al., 2004). Contudo, além da interpretação visual das imagens ser um processo extremamente tedioso e demorado, introduz uma subjetividade ao mapeamento da cultura, devendo ser utilizada de forma restrita, buscando-se, primeiramente, explorar as melhores alternativas da classificação digital.

Uma dessas alternativas pode ser o método de Superfícies de Resposta Espectro-Temporal (Spectral-Temporal Response Surface; STRS) (VIEIRA, 2000). A principal limitação da aplicação desse método para classificação de culturas agrícolas reside na dificuldade de se obter imagens livres de cobertura de nuvens, principalmente durante o ciclo das culturas de verão. Entretanto, a alta freqüência de aquisição das imagens do sensor MODIS MODerate-resolution Imaging Spectroradiometer (http://modis.gsfc.nasa.gov) tem propiciado a disponibilidade de um maior número de imagens livres de nuvens. Assim, estas imagens parecem ser uma opção interessante a ser explorada para fins de classificação da área de culturas agrícolas pelo método STRS. As imagens MODIS, sob a forma do produto MOD13Q1 (versão 4.0), são obtidas por meio da composição das melhores imagens com dados de reflectância de superfície, em termos de menor cobertura de nuvens, adquiridas ao longo de um período de 16 dias, sendo geométrica e radiometricamente corrigidas.
Apesar da alta resolução temporal diária das imagens MODIS, que propicia a obtenção de imagens livres de nuvens, a resolução espacial de 250 x 250m (6,25ha) apresenta limitações para o mapeamento, mesmo de grandes culturas, em especial em áreas de minifúndio(PAX-LENNEY \& WOODCOCK, 1997). Este trabalho tem por objetivo avaliar o potencial da classificação de áreas de soja no Estado do Rio Grande do Sul utilizando imagens de alta resolução temporal do sensor MODIS e o método para classificação de imagens multitemporais e multiespectrais STRS.

\section{MATERIAL E MÉTODOS}

A área de estudo está localizada na porção norte do Estado do Rio Grande do Sul, ocupando 1.562 .500 ha compreendidos entre $27^{\circ} 18^{\prime} 2^{\prime \prime}$ a $28^{\circ} 25^{\prime}$ $15^{\prime \prime}$ de latitude sul e entre $53^{\circ} 44^{\prime} 20^{\prime \prime}$ a $52^{\circ} 37^{\prime} 6^{\prime \prime}$ de longitude oeste (Figura 1). A área de estudo é caracterizada por regiões de topografia bastante acidentada, com áreas de preservação de vegetação secundária de remanescentes de floresta ombrófila mista (floresta de araucária) e de floresta estacional decidual (floresta tropical caducifólia) (IBGE, 2004). As áreas de topografia acidentada apresentam atividades agrícolas caracterizadas por minifúndios. Além da soja, o milho também possui certo destaque na região, utilizado geralmente em rotação com a soja para tornar mais eficiente o sistema denominado plantio direto na palha.

Um mapa temático da cultura da soja para esta região, referente à safra 2000/01, foi elaborado por RIZZI \& RUDORFF (2005), a partir de imagens multitemporais e multiespectrais dos sensores Thematic Mapper (TM) e Enhanced TM Plus (ETM+), a bordo dos satélites Landsat-5 e -7, respectivamente. Tal mapa foi reamostrado para a resolução espacial das imagens MODIS (250 x 250m) para ser considerado como mapa de referência, tanto na orientação da amostragem de pixels de treinamento para o classificador, quanto na posterior avaliação do resultado da classificação.

O critério de seleção das datas das imagens MODIS (produto MOD13Q1 versão 4.0) utilizadas teve por base, além do calendário agrícola da região e do ciclo fenológico da soja, a qualidade radiométrica das composições de imagens de 16 dias. A semeadura da soja no Rio Grande do Sul é recomendada de 10 de outubro a 20 de dezembro, com preferência para o mês de novembro. A colheita começa na primeira quinzena de março, com concentração maior no mês de abril (CUNHA et al., 2001). Estes períodos de concentração de semeadura e de colheita foram confirmados por RIZZI 


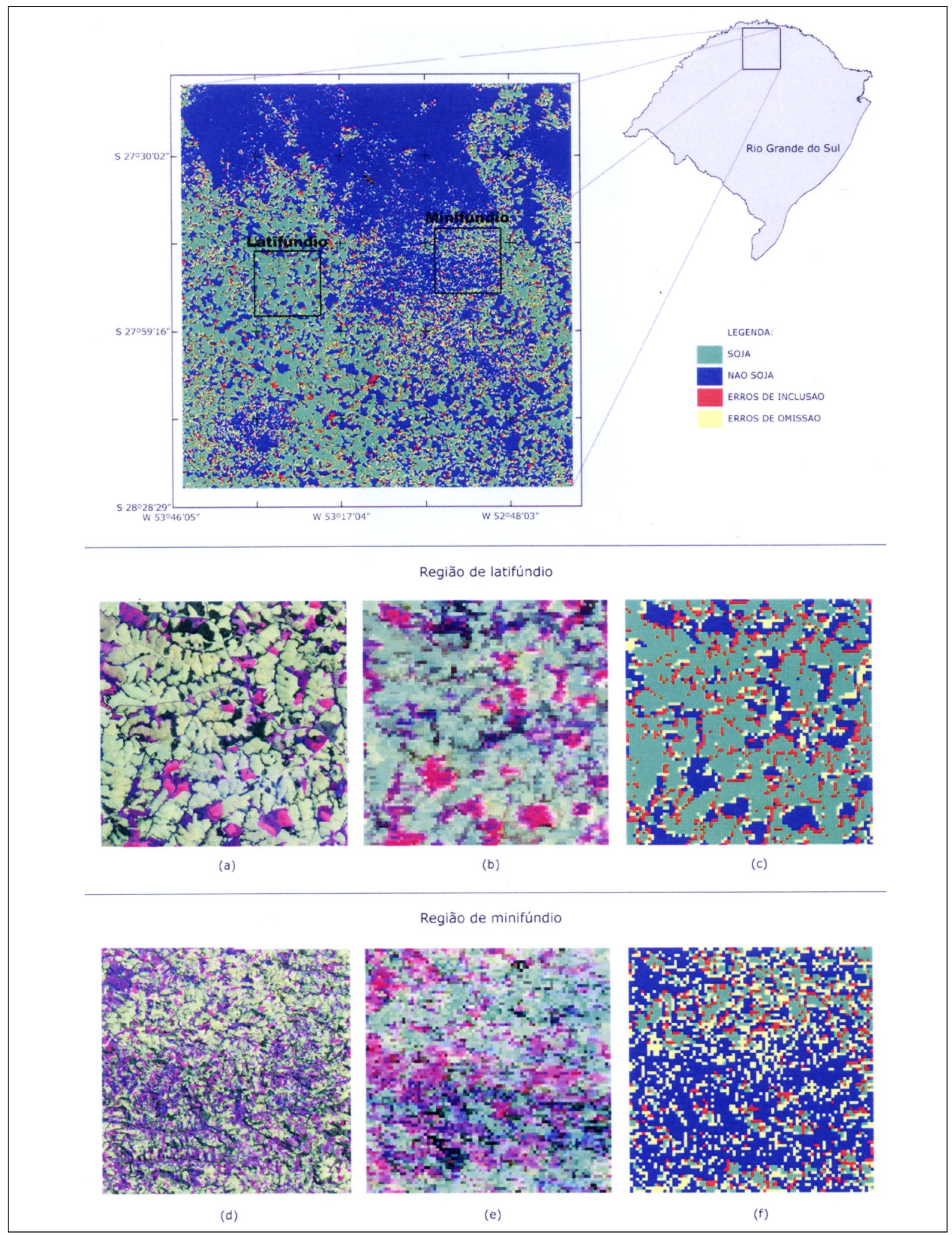

Figura 1 - No quadro superior, é apresentado o mapa resultante da classificação da soja pelo método STRS e os erros de inclusão e omissão para toda a área de estudo, (c) para a região de latifúndio e (f) para a região de minifúndio, safra 2000/01. (a) e (d) apresentam uma imagem do satélite Landsat-5, de 4 de março de 2001; (b) e (e) apresentam uma imagem MODIS do período de 18 de fevereiro a 05 de março de 2001.

Ciência Rural, v.37, n.1, jan-fev, 2007. 
(2004), que analisou a variação temporal dos índices de vegetação (Enhanced Vegetation Index; EVI e Normalized Difference Vegetation Index; NDVI) do MODIS (HUETE et al., 2002), ao longo da estação de crescimento da soja. Desta forma foi estabelecido que as imagens utilizadas neste trabalho fossem selecionadas dentro do período da segunda quinzena de outubro (semeadura) até a primeira quinzena de abril (colheita) da safra 2000/01, visando a abranger todo o ciclo da soja na região. Após uma avaliação visual da qualidade radiométrica das imagens abrangidas neste período, foram selecionadas seis composições de imagens de 16 dias para a classificação: de 15 a 30/10/ 2000, de 02 a 17/12/2000, de 18/02 a 05/03/2001, de 06 a 21/03/2001, de 22/03 a 06/04/2001 e de 07 a 22/04/2001. As bandas espectrais utilizadas foram: vermelho (banda 1), infravermelho próximo (banda 2) e infravermelho médio (banda 7).

A primeira imagem (de 15 a 30/10/2000) referese ao início da semeadura da soja. Portanto, observamse muitas áreas com solo exposto. Na imagem de 02 a 17 de dezembro de 2000, algumas áreas de soja já podem ser visualizadas. A imagem do período de 18 de fevereiro a 05 de março de 2001 é fundamental para a classificação da soja, pois, neste período, a maioria das lavouras se encontra em pleno desenvolvimento. Nas datas subseqüentes, dá-se o início da colheita, sendo que praticamente todas as áreas de soja estão em senescência ou colhidas na ocasião da imagem de 07 a 22 de abril de 2001.

O método STRS modela superfícies buscando caracterizar padrões de respostas espectrotemporais dos pixels em uma cena de imagens multiespectrais de diferentes datas. Este método foi proposto por VIEIRA (2000), em que cada pixel da imagem é representado em um espaço tridimensional com a distribuição das componentes temporal, espectral e radiométrica dispostas nos eixos $\mathrm{X}, \mathrm{Y}$ e $\mathrm{Z}$, respectivamente. Pontos de controle dos valores de intensidade de reflectância são posicionados em função do tempo, de acordo com as datas de aquisição das imagens na unidade de dias Julianos, e do espectro eletromagnético conforme o comprimento de onda central, em nanômetros, das diversas bandas do sensor.

Um método de interpolação é usado para parametrizar e gerar uma superfície analítica através dos pontos de controle, os quais podem ser irregularmente espaçados. VIEIRA (2000) testou dois métodos de interpolação para gerar as STRSs. O primeiro método é o de superfície de aproximação, conhecido como análise de tendência polinomial da superfície (Polynomial Trend Surface Analysis). Este método reduz os efeitos de pequenas variações nos pontos de controle. O segundo método é denominado Colocação, que proporciona superfícies fiéis aos pontos de controle por meio da combinação de três métodos de estimação: o ajustamento, a filtragem e a interpolação. O interpolador de superfície de aproximação pode ser vantajoso em casos de imagens com presença de nuvens ou de ruídos nos pixels. No entanto, considerando que os pixels das imagens MODIS (produto MOD13Q1 versão 4.0) apresentam-se livres de nuvens, utilizou-se neste trabalho o interpolador Colocação para gerar as superfícies STRS. Os detalhes e discussões sobre métodos de interpolação de superfícies podem ser encontrados em MATHER (1976).

Os coeficientes do polinômio parametrizado para cada superfície são extraídos para formar os atributos que serão adotados para o procedimento de classificação supervisionada. Desta forma, o aspecto multi-espectro-temporal considerado pelas STRS faz com que o espaço de atributos apresente uma estrutura diferente em relação à classificação elaborada com dados de imagens multiespectrais de única data. $\mathrm{Na}$ abordagem metodológica elaborada por VIEIRA (2000), a dimensionalidade do espaço de atributos corresponde ao número de coeficientes extraídos do polinômio.

O acréscimo de imagens temporais provoca um aumento de coeficientes das STRSs e, conseqüentemente, a dimensão do espaço de atributos considerado pelo classificador também aumenta. Este fato pode ocasionar problemas na classificação devido ao fenômeno conhecido como "Maldição da dimensionalidade" ("Hughes phenomenon”). Conforme demonstrado por VIEIRA (2000), a aplicação da técnica Forward Feature Selection sobre índices de separabilidade das classes permite reduzir a quantidade de coeficientes de entrada e melhorar a qualidade das estatísticas requeridas pelos classificadores paramétricos.

A figura 2 ilustra as etapas do procedimento envolvido na geração de um mapa temático a partir da classificação de imagens MODIS pelo método STRS. Conforme a configuração do conjunto de imagens MODIS utilizadas no presente trabalho, foram gerados 18 pontos de controle (3 bandas espectrais e 6 datas) tridimensionais para cada pixel (ver figura 2). Antes da interpolação das superfícies, os valores ao longo dos eixos X, Y e Z foram reescalonados para o intervalo de 0 a 1. Cada STRS foi gerada por meio do método de interpolação espacial Colocação, parametrizando uma função polinomial com 18 coeficientes.

As interpolações da variação espectrotemporal dos diversos alvos presentes na cena resultam 


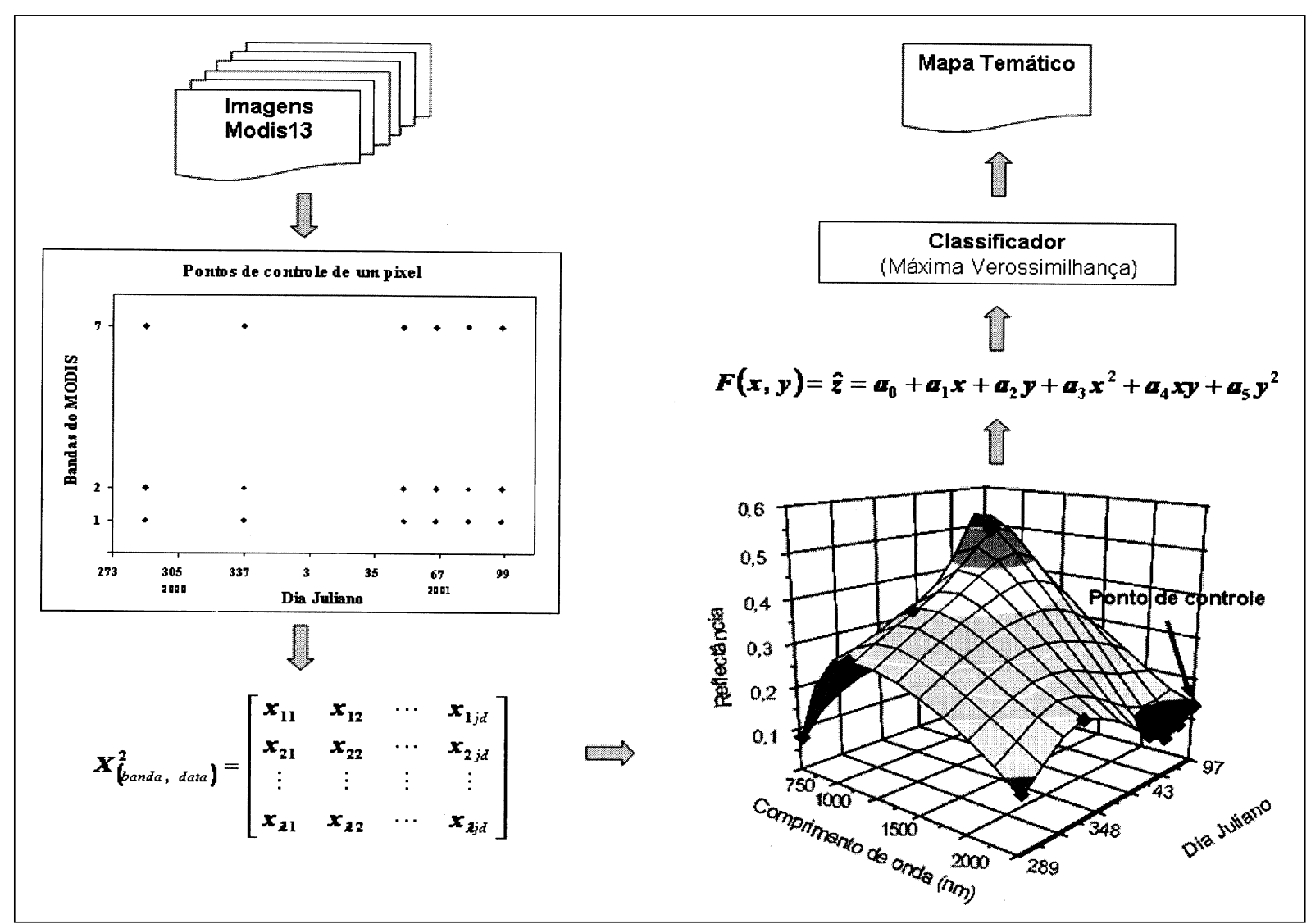

Figura 2 - Método STRS utilizado na classificação das áreas de soja sob imagens MODIS.

em superfícies com coeficientes de magnitudes muito variáveis. Portanto, antes de disponibilizar os coeficientes como parâmetros de entrada ao classificador, foi preciso escaloná-los para um intervalo entre 0 e 1, em relação aos valores máximos e mínimos encontrados entre os coeficientes de cada termo do polinômio para todos os pixels da cena.

A classificação foi realizada usando o algoritmo de Máxima Verossimilhança (MAXVER) adaptado ao espaço de atributos composto pelos coeficientes extraídos das STRSs. O MAXVER realiza a classificação de forma supervisionada, ou seja, requer um conhecimento a priori do número de classes de uso e cobertura do solo contidos em uma imagem. Assim, embora o objetivo da classificação tenha sido estimar a área de soja da safra 2000/01, foi preciso supervisionar o classificador considerando outros alvos e situações de uso do solo da região, visando a melhorar a separabilidade dos pixels de soja.

Para estabelecer os padrões das classes, foi necessário identificar um número suficiente de pixels na etapa de treinamento. Os pixels de treinamento da classe soja foram extraídos com base no mapa temático de referência mencionado anteriormente. Na área de estudo, foram observadas variações na data de semeadura da soja, decorrentes, possivelmente, do emprego de variedades de ciclo precoce, médio e tardio. As áreas de soja de semeadura média e tardia foram supervisionadas individualmente. Já as áreas de soja semeadas precocemente não puderam ser supervisionadas devido ao número limitado de áreas para o treinamento.

Os pixels de treinamento das demais classes consideradas (milho, floresta, pastagem, mancha urbana e água) foram extraídos com base nas imagens multitemporais do sensor MODIS e com o auxílio de uma imagem TM do satélite Landsat-5 de 4 de março de 2001. Para cada classe de treinamento, foram amostrados 250 pixels por meio de um procedimento de coleta aleatória estratificada (CONGALTON, 1991).

A fim de verificar o efeito da estrutura fundiária da produção de soja sobre o resultado da classificação, foi realizada ainda uma análise comparativa entre uma região de predomínio de lavouras de latifúndios e outra de minifúndios de soja, 
conforme ilustrado nas figuras 2a e 2d por meio de uma imagem TM adquirida em 04 de março de 2001.

O desempenho da classificação das áreas de soja, tanto para toda a área de estudo, quanto para as regiões de latifúndio e minifúndio, foi avaliado com base na exatidão global e nas exatidões do produtor e do usuário calculadas individualmente para cada classe. Os cálculos associados a estas medidas estão descritos em MATHER (1999) e foram realizados a partir da matriz de contingência (confusão). Para gerar cada matriz de contingência, foi utilizado um conjunto de 256 padrões amostrados por meio de procedimento de coleta aleatória estratificada.

O coeficiente Kappa ( $\mathrm{Ka}$ ) (CONGALTON, 1991) foi determinado para avaliar a significância do desempenho da classificação (representado pela matriz de contingência), por meio de um teste de hipótese que faz uma comparação entre as grandezas do mapa de referência e as do mapa de classificação. Estes resultados são resumidos na forma de uma matriz de significância, na qual os elementos da diagonal principal indicam a significância da classificação. A partir de cada matriz de contingência, o valor $\mathrm{Z}$ pode ser computado usando a fórmula $Z=K a / \operatorname{var}(K a)$, em que $Z$ é padronizado com distribuição normal e var é a variância do coeficiente Kappa. Se $Z \geq Z_{\alpha / 2}$, a classificação é significativamente melhor em relação a uma classificação aleatória, em que $\alpha / 2$ é o nível de confiança do teste $\mathrm{Z}$ e os graus de liberdade são assumidos como sendo infinitos. Por outro lado, os elementos fora da diagonal principal fornecem um indicativo, novamente se $Z \geq Z_{\alpha / 2}$, de que duas classificações independentes são significativamente diferentes. A equação usada para testar a significância da diferença entre dois coeficientes Kappa independentes é:

$$
Z=\left|K a_{1}-K a\right| / \sqrt{\left(\operatorname{var}\left(K a_{1}\right)+\operatorname{var}\left(K a_{2}\right)\right.}
$$

em que $K a_{1}$ e $K a_{2}$ são os dois coeficientes Kappa em comparação (CONGALTON \& GREEN, 1999).

\section{RESULTADOS E DISCUSSÃO}

A figura 1 (quadro superior) apresenta o resultado da classificação do método STRS para as classes soja e não-soja (demais classes presentes na região), os erros de omissão e inclusão para a classe soja, obtidos com base no mapa de referência descrito anteriormente, além da demarcação das regiões de latifúndio e minifúndio utilizadas para avaliação da classificação. A área de soja estimada pelo método STRS, para toda a região de estudo, foi de 574.687 ha, sendo subestimada em 6,6\%. Para a classe soja em toda a região, a exatidão do produtor foi de $65,74 \%$ e a exatidão do usuário foi de 74,74\% (Tabela 1). A exatidão

Tabela 1 - Matriz de contingência para toda a região, para a região de minifúndio e para a região de latifúndio.

\begin{tabular}{|c|c|c|c|c|c|}
\hline \multicolumn{6}{|c|}{ Toda a região } \\
\hline \multirow{6}{*}{ 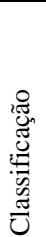 } & \multicolumn{5}{|c|}{ Referência } \\
\hline & Classe & Soja & Não-soja & Total & Exatidão usuário (\%) \\
\hline & Soja & 71 & 24 & 95 & 74,74 \\
\hline & Não-soja & 37 & 124 & 161 & 77,02 \\
\hline & Total & 108 & 148 & 256 & \\
\hline & Exatidão Produtor (\%) & 65,74 & 83,78 & & \\
\hline
\end{tabular}

\begin{tabular}{|c|c|c|c|c|c|}
\hline \multicolumn{6}{|c|}{ Latifúndio } \\
\hline \multirow{6}{*}{ 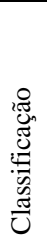 } & \multicolumn{5}{|c|}{ Referência } \\
\hline & Classe & Soja & Não-soja & Total & Exatidão usuário (\%) \\
\hline & Soja & 135 & 46 & 181 & 74,59 \\
\hline & Não-soja & 21 & 54 & 75 & 72,00 \\
\hline & Total & 156 & 100 & 256 & \\
\hline & Exatidão Produtor (\%) & 86,54 & 54,00 & & \\
\hline \multicolumn{6}{|c|}{ Minifúndio } \\
\hline \multirow{6}{*}{ 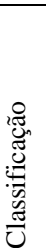 } & & & Referência & & \\
\hline & Classe & Soja & Não-soja & Total & Exatidão usuário (\%) \\
\hline & Soja & 41 & 15 & 56 & 73,21 \\
\hline & Não-soja & 56 & 144 & 200 & 72,00 \\
\hline & Total & 97 & 159 & 256 & \\
\hline & Exatidão Produtor (\%) & 42,27 & 90,57 & & \\
\hline
\end{tabular}


global em toda a região foi de 76,17\%, com valor da estatística Kappa de 0,503 (var = 0,003006251; estatística $Z=9,18$ ), que é considerado como bom desempenho (PAX-LENNEY \& WOODCOCK, 1997).

O resultado da classificação pelo método STRS sobre uma região de latifúndio e de minifúndio é apresentado nas figuras 1c e 1f, respectivamente. As estatísticas do teste de significância $\mathrm{Z}$ utilizando o coeficiente Kappa indicaram que o resultado da classificação para as regiões de latifúnio e minifundio foi significativo. O teste estatístico para verificar a significância das diferenças no desempenho da classifcação para as duas regiões indicou que não houve diferença significativa entre as classificações, muito embora na região de minifúndios a área de soja tenha sido subestimada em $43,4 \%$, enquanto que na região de latifúndios a área de soja foi superestimada em apenas 8\%.

Sobre a região de latifúndios, onde as lavouras são grandes e relativamente homogêneas, a exatidão global determinada foi de 74,83\%, com valor de estatística Kappa de 0,424 ( $\mathrm{Z}=7,33$ e var = $0,003354349)$. A exatidão do produtor para a classe soja na região de latifúndio foi de 86,54\% (Tabela 1). A maior parte dos erros de omissão foi observada para as lavouras de soja precoce, uma vez que o padrão espectro-temporal desta modalidade de plantio difere do padrão da soja média e tardia que, em sua maioria, foi amostrada para o treinamento do classificador. A exatidão do usuário para a classe soja na região de latifúndio foi de 74,59\% (Tabela 1). Os erros de inclusão tiveram maior predominância em lavouras de latifúnidos, pois muitos pixels da classe soja incluem, em menor proporção, outros alvos, como a mata ciliar que recorta as lavouras de soja (Figura 1e). Outra causa do erro de inclusão está ligada a uma situação de plantio de milho que apresenta um calendário agrícola semelhante ao da soja média. Apesar de a soja apresentar resposta espectral distinta do milho, nas bandas do infravermelho-próximo e-médio (RIZZI \& RUDORFF, 2005), não foi possível encontrar um número suficiente de pixels "puros” para representar a classe milho na etapa de treinamento.

Sobre a região de minifúndios, a baixa resolução espacial do sensor MODIS (250 x 250m $6,25 \mathrm{ha}$ ) faz com que os pixels da imagem freqüentemente sejam compostos por uma pequena parcela de soja, prevalecendo a resposta espectral das outras classes, resultando em erros de omissão. Já que a elevada mistura espectral da soja com outros alvos em um mesmo pixel impede o estabelecimento de STRSs semelhantes às definidas para um pixel "puro" de soja. A exatidão global determinada na região de minifúndio foi de 72,27\%, com valor de estatística Kappa de 0,358 $(\mathrm{Z}=6,13$ e var $=0,003402652)$. A exatidão do produtor para a classe soja foi de $42,27 \%$ (Tabela 1 ). A exatidão do usuário, na região de minifúndio, para classe soja, foi de 73,21\% (Tabela 1).

A região do Estado do Rio Grande do Sul que foi selecionada para a análise representa uma das situações mais complexas de distribuição espacial e tamanho das áreas de soja encontradas no Brasil. As lavouras estão freqüentemente situadas em minifúndios, o que dificulta sua identificação não por limitação do modelo STRS, mas pela resolução espacial insuficiente das imagens MODIS para detectar pequenas lavouras de soja. O método STRS aplicado às imagens multitemporais e multiespectrais do sensor MODIS mostrou-se eficiente para a classificação da soja em regiões com predominância de grandes lavouras, tal como são encontradas nas grandes regiões produtoras de soja do Brasil, como as regiões Centro-Oeste e Nordeste, onde a estrutura fundiária é quase que exclusivamente formada por latifúndios.

Um aspecto relevante no levantamento das estatísticas agrícolas é o conhecimento sobre a variação interanual da área plantada. O método proposto para classificação da soja pode prover informações sobre a localização de fronteiras agrícolas ou mesmo a intensidade da redução das áreas de plantio em razão de preços desfavoráveis para o setor. Estas informações podem ser utilizadas para auxiliar e aprimorar as estatísticas agrícolas convencionais e subjetivas.

Outro fato a ser destacado é que o método STRS empregado nas imagens MODIS pode fornecer informações a respeito da área plantada com soja em grandes regiões (nível estadual ou regional) de uma forma rápida, sem a necessidade da dispendiosa interpretação visual na tela do computador. Além disso, as imagens MODIS podem ser uma alternativa quando imagens de média resolução espacial (por exemplo, imagens Landsat) livres de cobertura de nuvens não estiverem disponíveis.

\section{CONCLUSÃO}

O método de classificação STRS, aplicado às imagens multiespectrais e multitemporais do sensor MODIS, demonstrou ter potencial para classificar e estimar a área de soja, principalmente em regiões predominantemente de latifúndios. Em regiões de minifúndios, a correta identificação e classificação das áreas de soja mostrou-se pouco eficiente, principalmente em função da resolução espacial insuficiente das imagens MODIS para identificar pequenas lavouras de soja.

Ciência Rural, v.37, n.1, jan-fev, 2007. 


\section{REFERÊNCIAS}

CONGALTON, R.G. A review of assessing the accuracy of classifications of remotely sensed data. Remote Sensing of Environment, v.37, n.1, p.35-46, 1991.

CONGALTON, R.G.; GREEN, K. Assessing the accuracy of remotely sensed data: principles and practices. New York: Lewis Publishers, 1999. 137p.

CUNHA, G.R. et al. Zoneamento agrícola e calendário de semeadura para soja no Rio Grande do Sul, safra 2001/ 2002. 2001. Acessado em 26 de ago. 2005. Online. Disponível na internet: http://www.cnpt.embrapa.br/biblio/ p_co80.htm\#endereço

HUETE, A. et al. Overview of the radiometric and biophysical performance of the MODIS vegetation indices. Remote Sensing of Environment, v.83, p.195-213, 2002.

IBGE Mapa de vegetação do Brasil 1:5.000.000, 2004. Acessado em 27 de ago. 2005. Online. Disponível na internet: ftp://ftp.ibge.gov.br/Cartas_e_Mapas/Mapas_Murais/ vegetação_pdf.zip

IPPOLITI-RAMILO, G.A. et al. Landsat-5 Thematic Mapper data for pre-planting area evaluation in tropical countries. International Journal of Remote Sensing, v.24, n.7, p.1521-1534, 2003.

MATHER, P.M. Computational methods of multivariate analysis in physical geography. Chichester: John Wiley \& Sons, 1976. 532p.
MATHER, P.M. Computer processing of remotely-sensed images: an introduction. 2.ed. Chichester: John Wiley \& Sons, 1999. 292p.

PAX-LENNEY, M.; WOODCOCK, C.E. The effect of spatial resolution on the ability to monitor the status of agricultural lands. Remote Sensing of Environment, v.61, n.2, p.210220, 1997

RIZZI, R. Geotecnologias em um sistema de estimativa da produção de soja: estudo de caso no Rio Grande do Sul. 2004. 214f. (INPE-12271-TDI/983). Tese (Doutorado em Sensoriamento Remoto) - Instituto Nacional de Pesquisas Espaciais, São José dos Campos. Acessado em 25 de ago. 2005. Online. Disponível na biblioteca digital URLib: http:// iris.sid.inpe.br:1912/rep-/sid.inpe.br/jeferson/2004/07.02.14.17

RIZZI, R.; RUDORFF, B.F.T. Estimativa da área de soja no rio grande do sul por meio de imagens landsat. Revista Brasileira de Cartografia, v.57, n.3, p.226-234, 2005.

RUDORFF, B.F.T. et al. Estimativa de área plantada com cana-de-açúcar em municípios do Estado de São Paulo por meio de imagens de satélites e técnicas de geoprocessamento: ano safra 2004/2005. São José dos Campos: INPE, 2004. 54p. (INPE-11421-RPQ/762).

VIEIRA, C.A.O. Accuracy of remotely sensing classification of agricultural s: a comparative study. 2000. 352f. Thesis (Degree of Doctor of Philosophy) - University of Nottingham; Nottingham. 